\title{
Prostacyclin promotes oligodendrocyte precursor recruitment and remyelination after spinal cord demyelination
}

\author{
C Takahashi ${ }^{1,2}$, R Muramatsu ${ }^{\star, 1,2}$, H Fujimura ${ }^{3}$, H Mochizuki ${ }^{2,4}$ and T Yamashita ${ }^{*, 1,2}$
}

Adult oligodendrocyte precursor cells (OPCs) are located adjacent to demyelinated lesion and contribute to myelin repair. The crucial step in remyelination is the migration of OPCs to the demyelinated area; however, the mechanism of OPC migration remains to be fully elucidated. Here we show that prostacyclin (prostaglandin $\mathrm{I}_{2}, \mathrm{PGI}_{2}$ ) promotes OPC migration, thereby promoting remyelination and functional recovery in mice after demyelination induced by injecting lysophosphatidylcholine (LPC) into the spinal cord. Prostacyclin analogs enhanced OPC migration via a protein kinase A (PKA)-dependent mechanism, and prostacyclin synthase expression was increased in the spinal cord after LPC injection. Notably, pharmacological inhibition of prostacyclin receptor (IP receptor) impaired remyelination and motor recovery, whereas the administration of a prostacyclin analog promoted remyelination and motor recovery after LPC injection. Our results suggest that prostacyclin could be a key molecule for facilitating the migration of OPCs that are essential for repairing demyelinated areas, and it may be useful in treating disorders characterized by demyelination.

Cell Death and Disease (2013) 4, e795; doi:10.1038/cddis.2013.335; published online 12 September 2013

Subject Category: Neuroscience

Oligodendrocytes form myelin sheaths around axons that are essential for the high-speed transmission of electrical impulses $^{1}$ and for providing trophic support to axons. ${ }^{2}$ Damage to oligodendrocyte lead to impairments in the motor, sensory, cognitive, and other neuronal functions. ${ }^{3}$ Multiple sclerosis (MS) is associated with multifocal demyelination and oligodendrocyte injury in the central nervous system (CNS). The proposed mechanisms of demyelination in MS vary depending on disease stage. During early stages, new myelin forms spontaneously in response to myelin degradation. ${ }^{4,5}$ The new myelin forms a thin sheath with short internodes that contribute to restoring rapid saltatory conductance in the CNS and preventing axonal damage. ${ }^{6}$ In the late chronic stages, remyelination failure is a prominent pathologic feature that probably contributes to the relentless progression of the disease. ${ }^{7}$ Therefore, determining how remyelination occurs in white matter under pathologic conditions may identify new therapeutic avenues to explore to treat CNS disease.

Myelination is mediated by oligodendrocyte precursor cells (OPCs) that are widely distributed throughout both the gray and white matter of the CNS throughout life. ${ }^{8,9}$ Adult-born oligodendrocyte can continue to proliferate and produce compact myelin. $^{9}$ In response to demyelinating insults, A2B5-positive $\left(\mathrm{A}^{2} \mathrm{~B}^{+}\right)$OPCs subsequently proliferate, migrate, and attain maturity to become $\mathrm{O} 4^{+}$premyelinating oligodendrocyte before differentiating into mature myelin basic protein (MBP)-positive myelinating oligodendrocytes in the demyelinated lesion. OPCs that are recruited to the lesioned area differentiate into mature remyelinating oligodendrocyte and are engaged in the formation of new myelin sheaths around axons. ${ }^{10}$ However, demyelinated MS plaques vary with regard to oligodendrocyte content, ${ }^{11}$ and some are characterized by a lack of OPCs, which is considered to be due to impaired recruitment of OPCs to demyelinated areas. ${ }^{7}$ To answer this significant question, it is important to elucidate the mechanism of OPC recruitment in the adult CNS.

Prostacyclin (prostaglandin $\mathrm{I}_{2}, \mathrm{PGI}_{2}$ ) is a predominant cyclooxygenase metabolite of arachidonic acid synthesized by vascular endothelial and smooth muscle cells. Prostacyclin acts as primary vasodilator as well as an inhibitor of leukocyte adhesion and platelet aggregation. ${ }^{12,13}$ Recent studies have emphasized that prostacyclin is not solely a regulator of the cardiovascular system; it is also involved in a diverse set of functions under homeostatic and pathological conditions in

\footnotetext{
${ }^{1}$ Department of Molecular Neuroscience, Graduate School of Medicine, Osaka University, Suita, Osaka 565-0871, Japan; ${ }^{2}$ Japan Science and Technology Agency, Core Research for Evolutional Science and Technology, Chiyoda, Tokyo 102-0075, Japan; ${ }^{3}$ Toneyama National Hospital, Toyonaka, Osaka, Japan and ${ }^{4}$ Department of Neurology, Graduate School of Medicine, Osaka University, Suita, Osaka, Japan

${ }^{*}$ Corresponding authors: R Muramatsu or T Yamashita, Department of Molecular Neuroscience, Graduate School of Medicine, Osaka University, 2-2 Yamadaoka, Suita, Osaka 565-0871, Japan. Tel: 81-6-68793661; Fax: 81-6-68793669; E-mail: muramatsu@ molneu.med.osaka-uac.jp or yamashita@ molneu.med.osaka-u.ac.jp Keywords: lysophosphatidylcholine; protein kinase A; multiple sclerosis

Abbreviations: OPC, oligodendrocyte progenitor cell; $\mathrm{PGI}_{2}$, prostacyclin $\mathrm{I}_{2}$; $\mathrm{MS}$, multiple sclerosis; CNS, central nervous system; MBP, myelin basic protein; DMEM, Dulbecco's Modified Eagle medium; PLL, poly-L-lysine; PDGF, platelet-derived growth factor; PFA, paraformaldehyde; PBS, phosphate-buffered saline; PKA, protein kinase A; PGIS, prostacyclin synthase; Th, thoracic; DAPI, 4',6-diamidino-2-phenylindole; BMS, basso mouse scale; SEM, standard error of the mean; ANOVA, analyses of variance; CAMP, cyclic adenosine monophosphate; IL, interleukin; IFN, interferon; EAE, experimental autoimmune encephalomyelitis; cPLA2 $\alpha$, cytosolic phospholipase A2 $\alpha$; mPGES-1, microsomal prostaglandin E synthase-1

Received 15.7.13; accepted 07.8.13; Edited by A Verkhratsky
} 

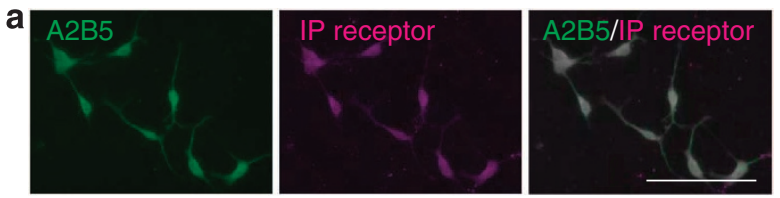

b IP receptor (67 kDa)

$\alpha$-Tubulin

(55 kDa)

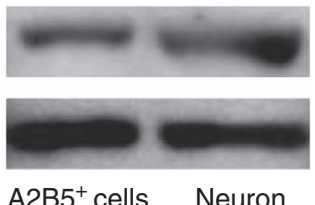

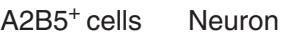

C

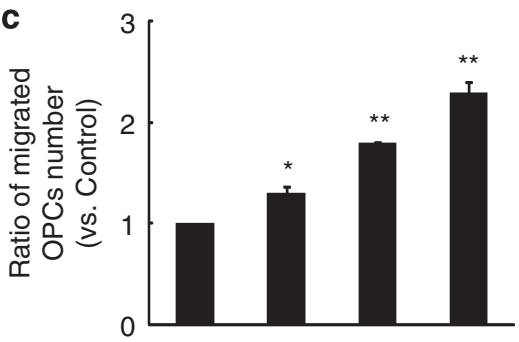

lloprost $(\mu \mathrm{M}) \quad 0$

e

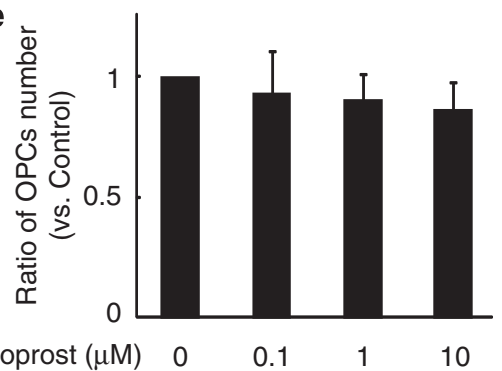

d

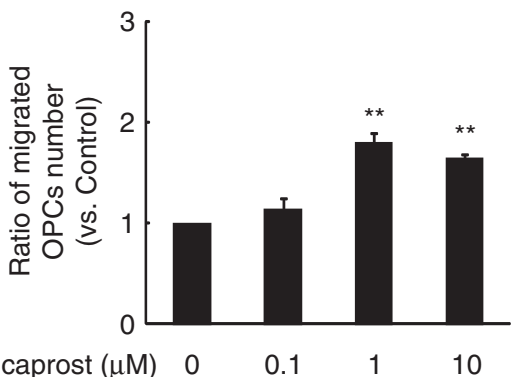

f

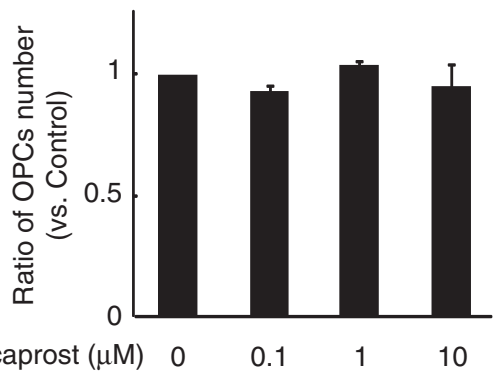

Figure 1 Prostacyclin analogs promote OPC migration in vitro. (a) Expression of IP receptor in A2B5 ${ }^{+}$OPC obtained from a postnatal day 1 mouse. Scale bar, $100 \mu \mathrm{m}$. (b) Western blot analyses for IP receptor (upper panel) and $\alpha$-tubulin (lower panel) in cultured OPCs (left) and cortical neurons (right). (c and d) Cell migration assay showing the dose response to prostacyclin analogs in OPCs incubated with the indicated concentration of iloprost (prostacyclin analog) (c) or cicaprost (prostacyclin analog) (d). The graphs show the relative number of cells that migrated to the lower chamber after $16 \mathrm{~h}$ of culture. (e and $\mathrm{f}$ ) The number of OPCs that were cultured under indicated conditions as described in Figures $1 \mathbf{c}$ and $\mathbf{d}$. Values represent the mean \pm S.E.M. $(n=3) .{ }^{*} P<0.05$ and ${ }^{* \star} P<0.01$ compared with control with individual prostacyclin analogs

the CNS. Both prostacyclin synthase (PGIS) and prostacyclin receptor are widely distributed in the CNS, ${ }^{14,15}$ and prostacyclin signaling has been demonstrated to protect neurons against ischemic damage. ${ }^{16,17}$ We previously showed that prostacyclin derived from neovessels accelerates axonal rewiring, thereby enhancing motor recovery following CNS injury. ${ }^{18}$ These findings prompted us to test the possibility that prostacyclin contributes to CNS remyelination caused by demyelination disorders.

Here, we studied the role of prostacyclin in OPC recruitment into demyelinated lesions. An in vitro migration assay revealed that prostacyclin promoted OPC migration without inducing cell proliferation. In the lysophosphatidylcholine (LPC)-induced focal spinal cord demyelination mouse model, protein expression of PGIS was increased. Treatment with IP receptor antagonist prevented both spontaneous OPC recruitment into the lesion site and functional recovery following LPC injection. Furthermore, treatment with a prostacyclin analog promoted OPC recruitment and improved demyelination-induced motor disability. Thus, prostacyclin could be a player in OPC recruitment and may serve as a promising molecule to treat demyelination diseases.

\section{Results}

Prostacyclin promotes OPC migration in vitro. We first examined the expression of prostacyclin receptor (IP receptor) in OPCs. We purified the A2B5 ${ }^{+}$OPCs from postnatal day 1 mice. Immunocytochemical analysis demonstrated that IP receptor was expressed in $\mathrm{A} 2 \mathrm{~B} 5{ }^{+} \mathrm{OPCs}$, which were cultured for 2 days in vitro (Figure 1a). IP receptor expression was also confirmed by western blotting of lysates from both OPC and cortical neuron (positive control) (Figure 1b). We then assessed whether IP receptor expression is associated with OPC migration. OPCs were plated onto the transwell culture insert containing stable prostacyclin analog, iloprost or cicaprost. After $16 \mathrm{~h}$, we counted the number of OPCs that migrated across the transwell membranes and determined 

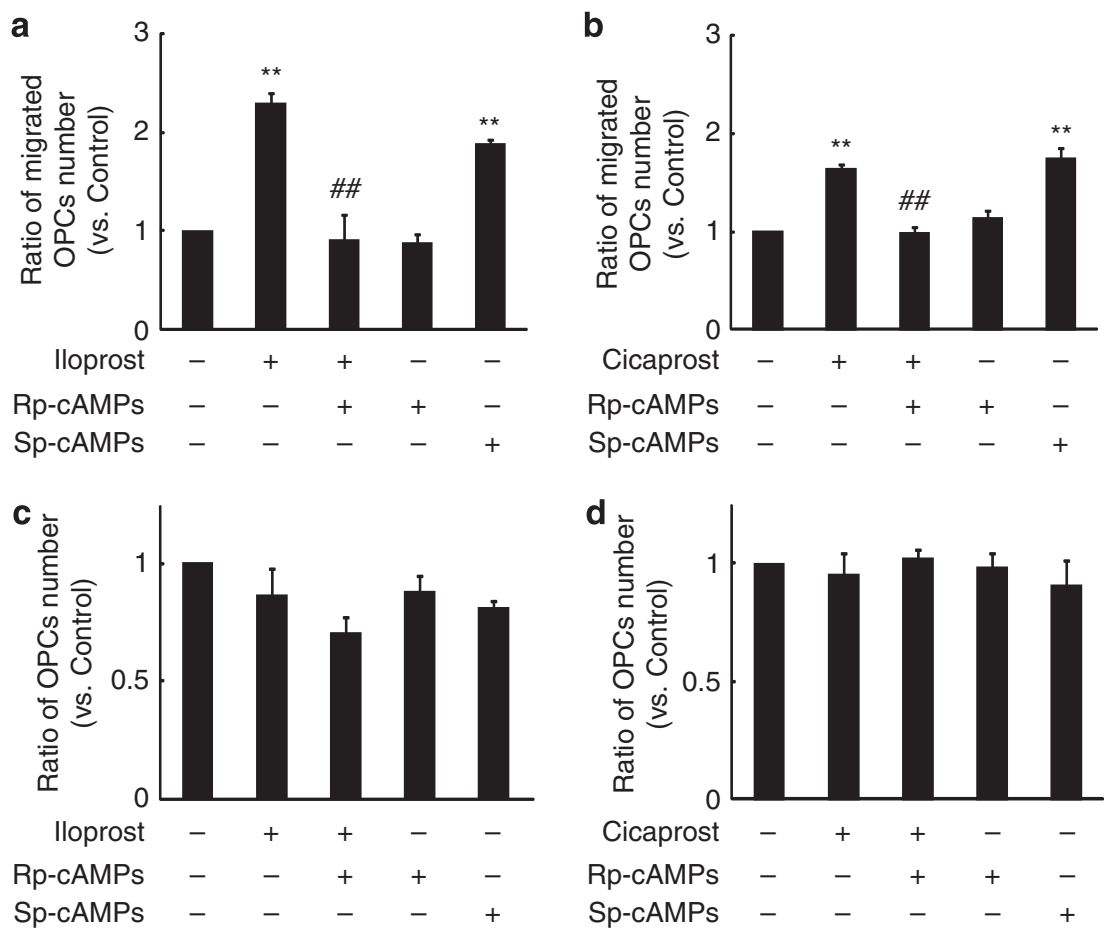

Figure 2 cAMP and PKA signal are required for prostacyclin-induced OPC migration. (a and $\mathbf{b})$ Quantification of the OPC migration under each treatment. Cells were preincubated with Rp-cAMPS, which is a PKA antagonist $(100 \mu \mathrm{M})$ and then cultured with iloprost ( $1 \mu \mathrm{M}$, prostacyclin analog) or cicaprost $(1 \mu \mathrm{M}$, prostacyclin analog). Sp-cAMPS is a PKA agonist $(100 \mu \mathrm{M})$. (c and $\mathbf{d})$ The graph shows the relative number of OPCs that were treated with indicated agents as described in Figures $2 \mathbf{a}$ and $\mathbf{b}$. Values represent the mean \pm S.E.M. $(n=3)$. ${ }^{\star *} P<0.01$ compared with control, ${ }^{\# \#} P<0.01$ compared with iloprost or cicaprost treatment

that the prostacyclin analogs promoted OPC migration (Figures 1c and d). To exclude the possibility that the increased number was due to increased cell proliferation, we counted the total number of OPCs in the cultures. There was no significant difference in cell number under any of the treatments (Figures $1 e$ and $f$ ). These results demonstrated that iloprost and cicaprost enhanced OPC migration in culture.

PKA is required for prostacyclin-induced OPC migration. IP receptor is a $\mathrm{G}$ protein-coupled receptor that stimulates adenylyl cyclase to synthesize adenosine $3^{\prime} 5^{\prime}$-monophosphate (cyclic adenosine monophosphate, cAMP) from adenosine triphosphate. ${ }^{19}$ Because $\mathrm{CAMP}$ elevation and protein kinase $A$ (PKA) activation have been shown to be required for efficient cell migration, ${ }^{20}$ we predicted that prostacyclin-mediated OPC migration would be dependent on PKA activation. Treatment with the PKA antagonist Rp-cAMPS abolished prostacyclin analog-mediated OPC migration (Figures $2 \mathrm{a}$ and b). Conversely, treatment with Sp-cAMPS, a PKA agonist, enhanced OPC migration (Figures $2 a$ and $b$ ). These pharmacological treatments did not alter the rates of OPC proliferation compared with control (Figures $2 \mathrm{c}$ and $\mathrm{d}$ ). The results suggest that PKA activation is required for OPC migration mediated by IP receptor signaling.

Expression of prostacyclin and its receptor in the adult mouse CNS and MS patient brains. To test whether our in vitro observations reflected the situation in vivo, we examined the role of prostacyclin in OPC accumulation in the LPC-induced demyelination model. Stereotaxic injection of LPC into the defined adult spinal cord results in selective and focal myelin loss without producing significant damage in adjacent cells and axons ${ }^{21,22}$ and induces subsequent remyelination within 4 weeks. ${ }^{21}$ During the remyelination process, OPCs are recruited toward demyelinated lesions. We hypothesized that this recruitment is promoted by prostacyclin. To test this hypothesis, we first investigated whether LPC injection into the spinal cord altered PGIS expression. In this model, we observed the complete ablation of resident OPCs at the lesion site 3 days after LPC injection (data not shown). Western blot analysis revealed that PGIS expression in the spinal cord tissue of LPC-injected mice increased compared with that in the control spinal cord tissue (Figure 3a). Immunohistochemical analysis demonstrated that majority of PDGFR $\alpha^{+}$OPCs expressed IP receptor in the spinal cord (dorsal column; Figure $3 b$ ). Moreover, we detected IP receptor expression in PDGFR $\alpha^{+}$cells in brain autopsy samples obtained from three individuals with MS (Figure 3c).

Prostacyclin promotes OPC recruitment and remyelination. To determine whether prostacyclin has a crucial role in OPC recruitment and remyelination, we administered the IP receptor antagonist CAY10441 via osmotic mini-pumps with intrathecally placed catheters into the lesion site 3 days after LPC injection. We counted the number of PDGFR $\alpha / 4^{\prime}$, 6-diamidino-2-phenylindole (DAPI) double-positive OPCs in the dorsal column of the spinal cord 7 days after LPC injection (Figure 4a). The number of accumulated OPCs was significantly attenuated in CAY10441-treated mice 


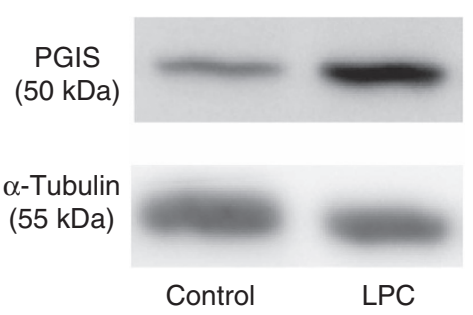

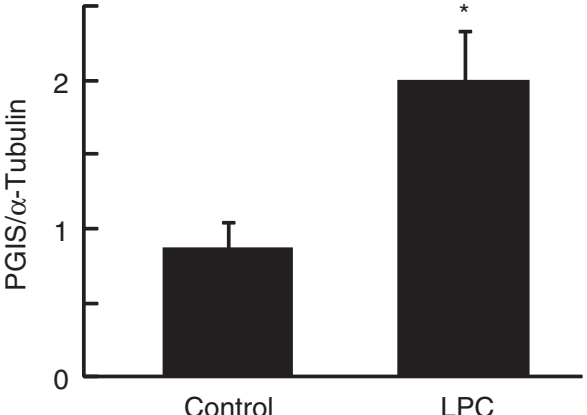

Control
LPC

b
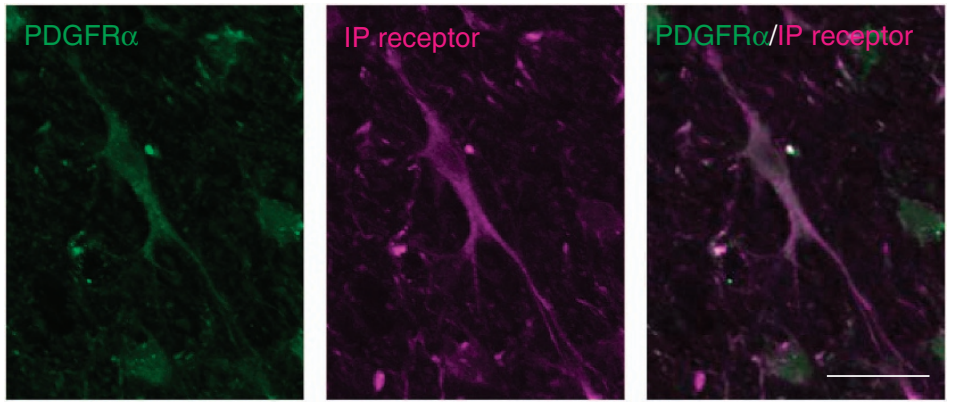

C
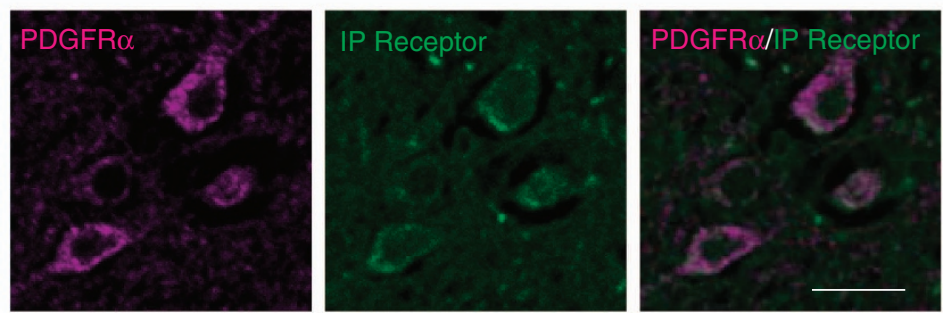

Figure 3 Prostacyclin and its receptor are expressed in the CNS tissue of mouse and MS patients. (a) Representative spinal cord sections immunostained for IP receptor (magenta) and PDGFR $\alpha$ (green) in control mice. Scale bar, $20 \mu \mathrm{m}$. (b) Representative brain section immunostained for IP receptor (green) and PDGFR $\alpha$ (magenta) in MS patients. Scale bar, $10 \mu \mathrm{m}$. (c) Western blot analyses for PGIS and $\alpha$-tubulin expression in the spinal cord. Spinal cord tissues were obtained 4 days after LPC injection. PGIS level was normalized to that of $\alpha$-tubulin. Values represent the mean \pm S.E.M. $(n=6) .{ }^{*} P<0.05$ compared with control

compared with vehicle-treated mice (Figures $4 \mathrm{~b}$ and c). As these accumulated OPCs are considered to contribute to subsequent remyelination, we investigated whether the inhibition of prostacyclin signaling also prevented remyelination after LPC injection. We assessed the expression of MBP, a major component of CNS myelin, in the dorsal column of the spinal cord 14 days after LPC injection. Immunohistochemistry showed that the extent of MBP distribution in the spinal cord was lower in CAY10441-treated mice compared with control mice (Figures $4 d$ and e). These results suggest that endogenous prostacyclin signaling is required for OPC recruitment and remyelination in the adult spinal cord.

We further examined whether prostacyclin can enhance OPC actions in the injured spinal cord. Intrathecal administration of iloprost facilitated OPC accumulation and myelin formation (Figures $4 \mathrm{c}-\mathrm{e}$ ), suggesting that it has promising therapeutic potential owing to its remyelinating properties.

Prostacyclin improves motor function after demyelinating insult. Finally, we assessed whether prostacyclin promoted restoration of neurological function by examining motor recovery after LPC-induced demyelination. Although focal demyelination in the dorsal column of the rodent spinal cord causes motor deficits, this impairment is recovered spontaneously when the area is remyelinated. ${ }^{22,23}$ We used two behavioral tests to evaluate impairment and subsequent recovery of hindlimb motor function: behavioral test recording (Figure 5a) and the basso mouse scale (BMS) (Figure 5b). Severe hindlimb impairment resulting from LPC injection was followed by spontaneous recovery up to day 28 after injection (Figures $5 \mathrm{a}$ and $\mathrm{b}$ ). Consistent with the histological results, treatment with CAY10441 significantly prevented spontaneous recovery of hindlimb function after LPC injection (Figures $5 \mathrm{a}$ and $\mathrm{b}$ ), suggesting that endogenous prostacyclin contributes to the spontaneous recovery of motor function. We then investigated the therapeutic effect of prostacyclin on motor deficits associated with demyelination and found that iloprost administration significantly promoted the recovery of motor function. This significant difference compared with vehicle control persisted from day 7 after LPC injection to day 14. These results demonstrate that prostacyclin has therapeutic potential for treating neurological dysfunction that is the result of demyelination. 

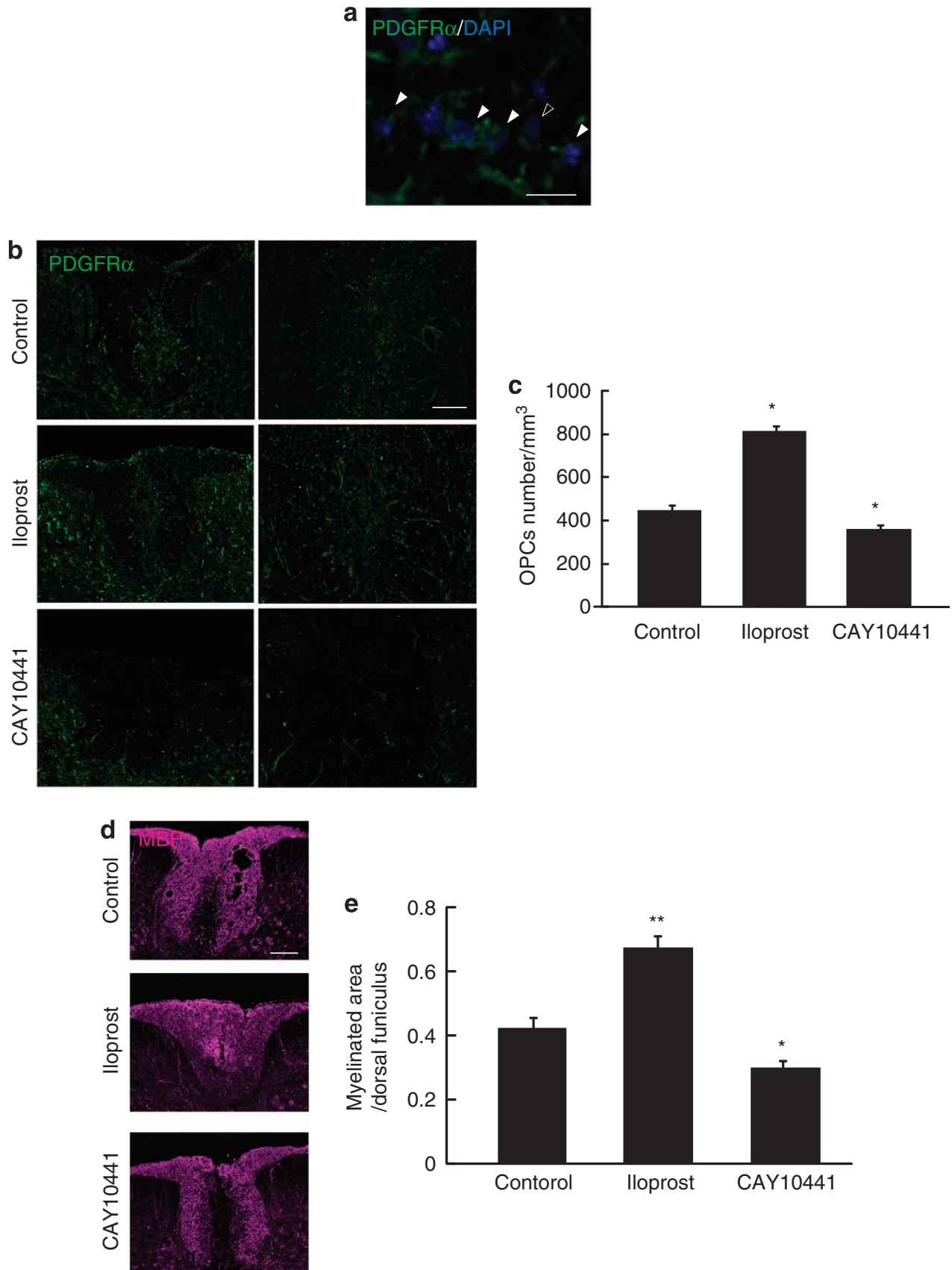

Figure 4 Prostacyclin drives OPC accumulation and remyelination after LPC-induced demyelination in the spinal cord. (a) Representative spinal cord sections doublelabeled for PDGFR $\alpha$ and DAPI. Arrowheads indicate OPCs, and the outlined arrowhead indicates a non-OPC in the dorsal funiculars of the spinal cord. Scale bar, $10 \mu \mathrm{m}$. (b) Representative spinal cord sections showing PDGFR $\alpha$-positive OPCs 7 days after LPC injection with or without IP receptor antagonist CAY10441 or iloprost treatment. Scale bars, $50 \mu \mathrm{m}$. (c) Quantification of accumulated OPCs in the spinal cord 7 days after LPC injection. Values represent the mean \pm S.E.M. ( $n=5$ ). (d) Representative spinal cord sections showing MBP-positive myelin 14 days after LPC injection and treatment with CAY10441 and iloprost. Scale bar, $100 \mu \mathrm{m}$. (e) Quantification of the MBP-positive myelinated area in the spinal cord 14 days after LPC injection. Values represent the mean \pm S.E.M. $(n=5) .{ }^{*} P<0.05$ and ${ }^{* *} P<0.01$ compared with control

Prostacyclin has been reported to be an inflammatory mediator that is associated with cytokine expression ${ }^{24,25}$ and inflammatory cell function. ${ }^{26}$ To test whether neurological recovery is independent of inflammation, we measured cytokine expression in spinal cord tissue treated with each pharmacological reagent. There were no differences in the amounts of the following cytokines: interleukin (IL)- $1 \alpha$, IL-1 $\beta$, IL-2, IL-3, IL-4, IL-5, IL-6, IL-10, IL-12p70, IL-17, interferon- $\gamma$, tumor necrosis factor- $\alpha$, monocyte chemoattractant protein-1, granulocyte-monocyte colony-stimulating factor, macrophage colony-stimulating factor, and regulated upon activation normal $T$ cell expressed and secreted released in the spinal cord tissue obtained from control and iloprost- or CAY10441-treated mice 4 days after LPC injection (data not shown). These data suggest that prostacyclin signaling did not affect inflammation at the demyelinating lesions. 

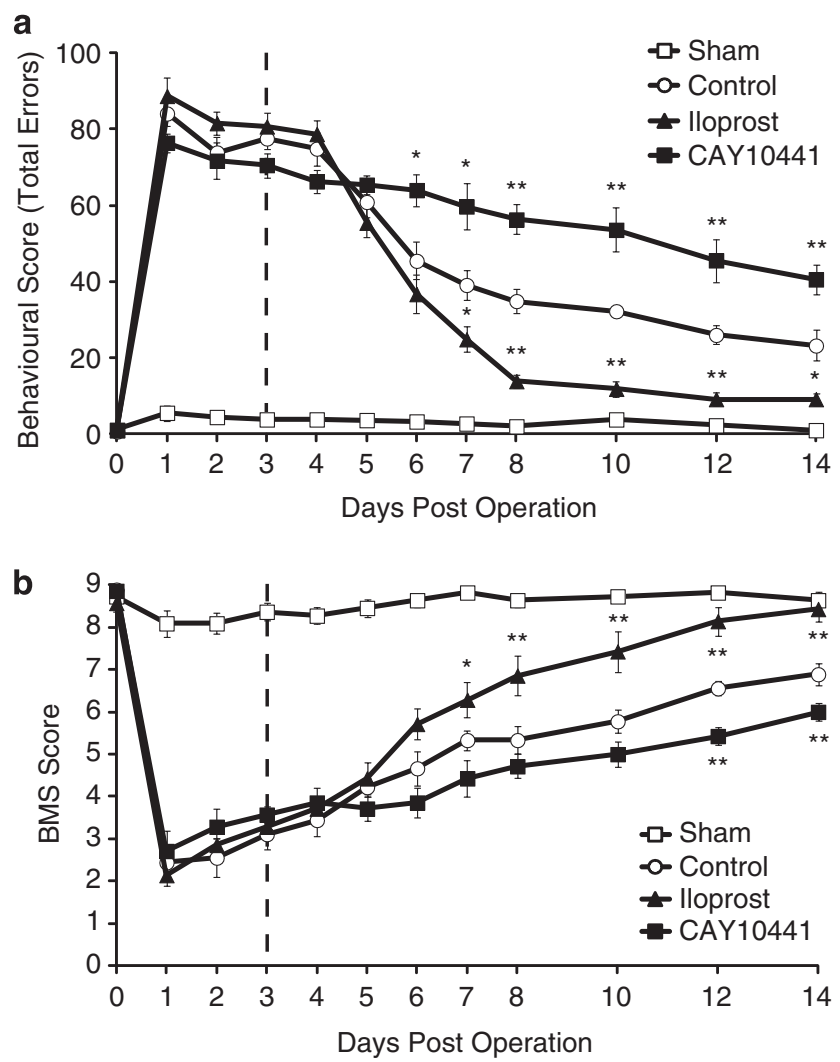

Figure 5 Prostacyclin promotes recovery from motor deficits caused by LPCinduced spinal cord demyelination. ( $\mathbf{a}$ and $\mathbf{b}$ ) The graphs depict the beam walking score (a) and BMS score (b) to estimate motor function. Values represent the mean \pm S.E.M. (control: $n=9$, sham: $n=11$, iloprost: $n=7$, and CAY10441: $n=7) .{ }^{*} P<0.05$ and ${ }^{* *} P<0.01$ compared with control

Taken together, our findings show that prostacyclin drives remyelination through OPC accumulation by activating IP receptor signaling. This effect appears to enhance hindlimb motor function recovery following localized demyelination induced by LPC injection.

\section{Discussion}

In this study, we elucidated the role of prostacyclin signaling in remyelination both in vitro and in vivo. The results of our in vitro migration assays demonstrate that prostacyclin promotes OPC migration via PKA signaling. In the commonly used model of LPC-induced demyelination of the dorsal column of the spinal cord, PGIS expression was upregulated. Treatment with IP receptor antagonist prevented spontaneous OPC accumulation and remyelination in the lesion after LPC injection. Conversely, prostacyclin analog enhanced OPC accumulation and remyelination. Finally, two behavioral analyses identified prostacyclin as the factor responsible for enhancing neurological recovery after demyelination.

The involvement of prostaglandins in MS pathogenesis was suggested by the finding that some prostaglandins were increased in the cerebrospinal fluid of MS patients. ${ }^{27}$ It is generally accepted that MS is an autoimmune disease characterized by immune activation and cell infiltration.
The role of prostaglandins in MS has been mainly studied with a focus on immunoregulation using the prevailing animal model of MS, experimental autoimmune encephalomyelitis (EAE). ${ }^{28}$ Cytosolic phospholipase A2 $\alpha$ (cPLA2 $\alpha$ ) is one of the critical enzymes involved in generating multiple prostaglandins. cPLA2 $\alpha$ selectively cleaves arachidonyl phospholipids to release free arachidonic acid, which is converted primarily to prostaglandins via the cyclooxygenase pathway. It is reported that $\mathrm{CPLA} 2 \alpha$-deficient mice, which cannot produce endogenous levels of PGs, are resistant to EAE induction. ${ }^{29}$ In addition, adoptive transfer experiments revealed that $\mathrm{CPLA} 2 \alpha$ is also involved in both the induction and the effector phases of EAE. ${ }^{29}$ There is ample evidence to suggest that $\operatorname{cPLA} 2 \alpha$ and its products are related to inflammation and autoimmune disease through PG production. This is in agreement with recent reports suggesting that $\mathrm{PGs}$, especially $\mathrm{PGE}_{2}$, has a pro-inflammatory role in EAE pathogenesis. ${ }^{30}$ Microsomal prostaglandin $\mathrm{E}$ synthase-1-deficient mice show less severe EAE symptoms and lower IL-17 and interferon- $\gamma$ production compared with control mice. ${ }^{31}$ Thus, PGs are considered to enhance inflammation and EAE severity. However, it is important to note that PGs may have other role(s) besides as an inflammatory factor. Thromboxane $A_{2}$, another arachidonic acid metabolite, is known to promote oligodendrocyte proliferation and survival. ${ }^{32}$ Oligodendrocytes are considered to contribute to spontaneous myelin repair, which is a characteristic feature in some acute MS lesions. ${ }^{4,5}$ In our study, prostacyclin facilitated spontaneous OPC recruitment, and this effect was independent of cytokine expression. This supports a previous report that prostacyclin fails to ameliorate EAE progression. ${ }^{25}$

Previous studies have reported that CAMP elevation enhances remyelination in the adult CNS. ${ }^{33,34}$ Relevant to remyelination, the adult OPCs undergo several developmental steps to become mature cells that initiate remyelination. Several studies have reported that CAMP analogs have been shown to induce the expression of myelin constituents in cultured oligodendrocytes, ${ }^{35,36}$ indicating that CAMP elevation induces OPC differentiation. On the other hand, OPC proliferation is not affected by cAMP level. ${ }^{37,38} \mathrm{PKA}$, the major target for cAMP action, is generally considered to induce the hallmarks of cell migration (e.g., actin filament assembly and activation of small $\mathrm{G}$ proteins). ${ }^{20}$ Indeed, our in vitro experiments showed that OPC migration upregulated by prostacyclin is dependent on PKA activation.

IP receptor is expressed in various mouse organs, including thymus, spleen, brain, and vascular tissues. ${ }^{14}$ The expression pattern is inferred to be critical for the development of the cardiovascular, immune, and nervous systems. However, IP receptor-deficient mice are viable, reproductive, and normotensive. Furthermore, no morphological or histological abnormalities were detected. $^{39}$ Meanwhile, IP receptordeficient mice showed slightly altered responses to acute nociceptive stimuli, ${ }^{39}$ suggesting that the role of endogenous prostacyclin-IP receptor signaling is restricted to pathological conditions. Indeed, we revealed that abundant prostacyclin expression in demyelinating lesion contributes to OPC recruitment through IP receptors, which is constitutively expressed on OPCs. Thus, lack of endogenous prostacyclin-IP receptor signaling might be compensated during the developmental stage and in physiological conditions, but 
endogenous prostacyclin-IP receptor signaling may be required for the neural network restoration under some pathological conditions.

Which cells produce prostacyclin in the diseased CNS? Angiogenesis, the formation of new blood vessels from an existing capillary, is concurrent with tissue repair and is essential for wound healing in adults. Prostacyclin is synthesized by vascular endothelial cells and smooth muscle cells. We recently reported that PGIS expression was increased in vascular endothelial cells in EAE mice. ${ }^{18}$ Therefore, vascular endothelial cells may be candidate cells for prostacyclin production.

Emerging data indicate that cell-cell interactions between the endothelium and neuronal cells helps sustain brain homeostasis and function. ${ }^{40-42}$ It is now well accepted that this can be partially explained by the ability of endothelial cells to produce trophic factors. Recent studies have shown that endothelium-derived factor(s) also act on OPCs, inducing their proliferation in vitro. ${ }^{43}$ Our findings support the hypothesis that an 'oligovascular niche' might also exist in the adult CNS and is partially regulated by prostacyclin. Importantly, this oligovascular niche may provide a promising new approach for treating demyelinating diseases.

\section{Materials and Methods}

Mice. C57BL/6 J mice were obtained from SLC Japan (Hamamatsu, Japan). All experimental procedures were approved by the Institutional Animal Care and Use Committee of Osaka University.

Primary culture of OPCs. OPCs were prepared from whole brains obtained from postnatal day 1 mice. We dissociated fresh mouse brain in Dulbecco's modified Eagle's medium (DMEM, Invitrogen, Carlsbad, CA, USA). After washing, we filtered the cell suspension through a $70-\mu \mathrm{m}$ cell strainer to obtain a single-cell suspension. Cells were incubated with A2B5-specific antibody-coated magnetic beads (Miltenyi-Biotec, Bergisch Gladbach, Germany) to isolate selectively OPCs. The isolated cells were plated a density of $4 \times 10^{4}$ cells/well into poly-L-lysinecoated four-well chamber slides (Nunc, Rochester, NY, USA) in DMEM containing $4 \mathrm{mM}$ L-glutamine (MP Biomedicals, Aurora, OH, USA), $1 \mathrm{mM}$ sodium pyruvate (Sigma, St.Louis, MO, USA), 0.1\% bovine serum albumin (BSA, Sigma), $50 \mu \mathrm{g} / \mathrm{ml}$ apo-transferrin (Sigma), $5 \mu \mathrm{g} / \mathrm{ml}$ insulin (Sigma), $30 \mathrm{nM}$ sodium selenite (Sigma), $10 \mathrm{nM}$ biotin (Sigma), $10 \mathrm{nM}$ hydrocortisone (Sigma), $10 \mathrm{ng} / \mathrm{ml}$ platelet-derived growth factor (PDGF)-AA (PeproTech, Rocky Hill, NJ, USA), and $10 \mathrm{ng} / \mathrm{ml}$ basicfibroblast growth factor (FGF) (PeproTech). Cells were maintained at $37^{\circ} \mathrm{C}$ and at $5 \% \mathrm{CO}_{2}$.

After culturing for $24 \mathrm{~h}$, the cells were fixed with $4 \%$ paraformaldehyde (PFA) in phosphate-buffered saline (PBS) for $30 \mathrm{~min}$ at room temperature. The cells were then permeabilized in PBS containing 5\% BSA and $0.1 \%$ Triton X-100 for $1 \mathrm{~h}$ at room temperature. Cells were double-labeled with mouse anti-A2B5 $(1: 300$, Millipore, Bedford, MA, USA) and rabbit anti-IP receptor (1:100, Cayman, Ann Arbor, MI, USA) diluted in PBS containing 5\% BSA and $0.1 \%$ Triton X-100 overnight at $4{ }^{\circ} \mathrm{C}$. The cells were visualized with secondary Alexa Fluor 488- or 568conjugated goat antibody to rabbit $\lg G$ and goat antibody to mouse $\operatorname{IgM}(1: 500$, Invitrogen). Images were acquired using an upright microscope and DP-controller image system (Olympus, Tokyo, Japan).

Primary culture of cortical neurons. Cortical neurons were prepared from cerebral cortices obtained from postnatal day 1 mice. The cerebral cortices were dissociated by trypsinization (treatment with $0.25 \%$ trypsin (Invitrogen) and $0.5 \mathrm{mg} / \mathrm{ml}$ DNase (Sigma) in PBS for $15 \mathrm{~min}$ at $37^{\circ} \mathrm{C}$ ) followed by resuspension in DMEM containing $10 \%$ fetal bovine serum (FBS, Invitrogen). The isolated cells were plated on poly-L-lysine-coated dishes at a density of $4 \times 10^{4}$ cells/well and maintained at $37^{\circ} \mathrm{C}$ and at $5 \% \mathrm{CO}_{2}$

Migration assay. OPC migration was examined in transwell cell-culture inserts (6.5-mm diameter, $8-\mu \mathrm{m}$ pore size, Corning Costar, Corning, NY, USA).
Cells were suspended in $10 \%$ FBS-DMEM at a concentration of $4 \times 10^{4}$ cells $/ \mathrm{ml}$. A $0.1 \mathrm{ml}$ cell suspension (final concentration: 4000 cells/well) was added to the upper compartment, and cells were then incubated for $16 \mathrm{~h}$. After fixation with $4 \%$ PFA, the transwell filters were incubated for $30 \mathrm{~min}$ in a solution of 4,6-diamidinophenylindole (DAPI, Santa Cruz, Santa Cruz, CA, USA) at the final concentration of $1 \mu \mathrm{g} / \mathrm{ml}$ in PBS. To avoid the observation of non-migrating OPCs, cells on the upper surface of the filter were removed by wiping with filter paper. Images of the bottom of the transwell filter were captured with an upright microscope using a $\times 4$ objective (Olympus). We counted the number of $\mathrm{DAPI}^{+}$cells and calculated the ratio of cultured cells treated with pharmacological agents to the number observed under control conditions. For pharmacological experiments, the following reagents were added into the lower compartment at the beginning of the culture period: Sp-cAMPS (PKA agonist, Sigma), Rp-cAMPS (PKA antagonist, Sigma), iloprost (a stable prostacyclin analog, Cayman Chemical), and cicaprost (another prostacyclin analog, Cayman Chemical).

Proliferation assay. OPCs were plated at a density of $8 \times 10^{4} \mathrm{cells} / \mathrm{ml}$ in DMEM containing $10 \%$ FBS with the indicated agents for $16 \mathrm{~h}$. Cell proliferation was estimated by counting the A2B5/DAPI double-positive cells. Images were captured with an upright microscope equipped with a $\times 4$ objective (Olympus). We calculated a ratio of cultured cells treated with pharmacological agents to the number observed under control conditions.

Western blotting. Cells or spinal cord tissues were lysed with RIPA buffer $(150 \mathrm{mM} \mathrm{NaCl}, 1.0 \% \mathrm{NP}-40,0.5 \%$ sodium deoxycholate, $0.1 \%$ sodium dodecyl sulfate, and $50 \mathrm{mM}$ Tris at $\mathrm{pH}$ 7.4) containing protease inhibitors (complete protease inhibitor cocktail, Roche Diagnostics, Mannheim, Germany). Cells or tissue lysates were then homogenized and centrifuged at $17400 \times g$ for $20 \mathrm{~min}$ at $4{ }^{\circ} \mathrm{C}$, and protein concentrations in the supernatants were determined with bicinchoninic acid protein assay (Thermo Scientific, Waltham, MA, USA). Proteins were separated by $7.5 \%$ SDS-polyacrylamide gel electrophoresis and transferred onto polyvinylidene difluoride membranes (Immobilon-P, Millipore). After blocking with PBS containing $5 \%$ skim milk and $0.05 \%$ Tween-20, the membranes were incubated with solutions of rabbit anti-IP receptor (1:500, Cayman Chemical), mouse anti- $\alpha$-tubulin (1:500, Santa Cruz), or rabbit anti-PGIS (1:100; Cayman Chemical) overnight at $4^{\circ} \mathrm{C}$. Membranes were incubated with horseradish peroxidase-conjugated secondary antibodies $(1: 5000$, Cell Signaling Technology, Danvers, MA, USA) for $1 \mathrm{~h}$ at room temperature. Signals were detected by an enhanced chemiluminescence (ECL) system (GE Healthcare, Munich, Germany), and protein expression quantification was performed using ImageJ software $(\mathrm{NIH}$; http://rsb.info.nih.gov/ij/).

LPC-induced demyelination in mouse spinal cord. Adult female mice were anesthetized with sodium pentobarbital before undergoing laminectomy at the thoracic level 11-12. We injected $2 \mu \mathrm{l} 1 \%$ LPC (Sigma) dissolved in saline into the dorsal column midline at a depth of $0.5 \mathrm{~mm}$.

For administration of pharmacological reagents, osmotic pump (model no. 1002 and no. 1007D; ALZET Corp., Cupertino, CA, USA) was filled with vehicle solution (saline), CAY10441 (IP receptor antagonist, $20 \mu \mathrm{g} / \mathrm{kg} /$ day; Cayman Chemical), or iloprost $(20 \mu \mathrm{g} / \mathrm{kg} /$ day, Cayman Chemical). The delivery tube connected to an osmotic pump was placed close to the lesion 3 days after LPC injection. The pump was implanted subcutaneously in the dorsal skin.

Immunohistochemistry of mouse tissue. Mice were transcardially perfused with 4\% PFA in PBS. The spinal cords were post-fixed with 4\% PFA in PBS for $24 \mathrm{~h}$ and then transferred sequentially into 20 and $30 \%$ sucrose solution until they were equilibrated. The spinal cords were sectioned in the transverse plane on a sliding microtome at $20 \mu \mathrm{m}$ and were mounted on Matsunami adhesive silane-coated Superfrost/Plus slides (Matsunami, Osaka, Japan). Five to seven sections were obtained from every $100 \mu \mathrm{m}$ that included the lesion epicenter.

For immunohistochemistry, sections were treated with $5 \%$ BSA plus $0.1 \%$ Triton $\mathrm{X}-100$ in PBS for $1 \mathrm{~h}$ at room temperature. The sections were incubated with primary antibodies diluted in PBS containing 5\% BSA and $0.1 \%$ Triton X-100 overnight at $4{ }^{\circ} \mathrm{C}$, followed by incubation with secondary antibodies for $1 \mathrm{~h}$ at room temperature. Images were taken by a fluorescence (Olympus BX51, DP71) or confocal laserscanning microscope (Olympus FluoView FV1000). The following primary antibodies were used: rat anti-CD140a (PDGFR $\alpha, 1: 50$; BD Biosciences, Erembodegem, Belgium), rabbit anti-IP receptor (1:100, Cayman Chemical), rat anti-MBP (1:50, Abcam, Cambridge, MA, USA). Secondary antibodies were Alexa 
Fluor 488- and 568-conjugated antibodies produced in goat (1:500, Invitrogen). Nuclear staining was performed with DAPI.

To assess OPC accumulation in the spinal cord, we counted the number of PDGFR $\alpha / D A P I$ double-positive cells in the dorsal column of spinal cord. We obtained the cell number of 10 areas $(50 \times 50 \times 20 \mu \mathrm{m})$ from $100-\mu \mathrm{m}$ intervals in the dorsal column of the spinal cord. For quantification of myelin formation, we measured the MBP-positive area in the dorsal column of the spinal cord.

Immunohistochemistry of human tissues. We obtained autopsied spinal cord tissue from three individuals with relapse-remitting MS. We fixed samples with formalin and then embedded the tissue in paraffin before preparing $4-\mu \mathrm{m}$-thick slices for immunohistochemistry. We deparaffinized sections with xylene followed by $100 \%$ ethanol. Antigen retrieval was performed by incubation at $98^{\circ} \mathrm{C}$ for $40 \mathrm{~min}$ in citrate buffer $(10 \mathrm{mM}, \mathrm{pH} 6.0)$. We incubated tissue samples with primary antibodies specific for human PDGFR $\alpha$ (1:100, R\&D Systems, Minneapolis, MN, USA) and IP receptor (1:100; Cayman Chemical). We used Alexa Fluor 488- and 568-conjugated antibodies (1:500, Invitrogen) for secondary antibodies. The research protocol was approved by the Human Use Review Committees of the Graduate School of Medicine, Osaka University, and Toneyama National Hospital, for the Protection of Human Subjects. Informed consent was obtained from all subjects.

Basso mouse scale. Recovery of hindlimb motor function was scored by the BMS open-field locomotor rating scale, which was developed specifically for mice. ${ }^{44}$ The score ranges from 0 (complete paralysis) to 9 (normal mobility), and the number of errors at each footstep was measured for 100 steps. Mice were observed individually for $2 \mathrm{~min}$ in an open field.

Behavioral data recording. Behavioral scoring was carried out while viewing the recording in slow motion on a standard video recorder. ${ }^{23}$ Scores were given to every step according to the flowing scheme: 0 , normal step; 1 , a minor error (slight insecurity of foot placement); or 2, major error (foot slipped completely from the beam surface). The total score for each step per mouse was used for subsequent statistical analysis.

Cytokine analysis. Spinal cord tissues at the level of Th11-12 were homogenized in $10 \mathrm{mM}$ Tris- $\mathrm{HCl}(\mathrm{pH} 7.4), 150 \mathrm{mM} \mathrm{NaCl}, 1 \%$ Triton X-100, and $1 \mathrm{mM}$ EDTA containing protease inhibitors. Samples were centrifuged at $17400 \times g$ for $10 \mathrm{~min}$ at $4^{\circ} \mathrm{C}$, and the supernatants were collected. The expression profiles of a panel of cytokines were measured using the Q-Plex Mouse Cytokine Array (Quansys Biosciences, Logan, UT, USA) according to the manufacturer's instructions.

Statistical analysis. Data are presented as mean \pm S.E.M. For the analysis of PGIS protein expression, differences between groups were examined using Student's $t$-tests. For behavioral analysis, differences between groups were examined using two-way repeated measure analyses of variance (ANOVAs) followed by post hoc Bonferroni tests. Other experiments were analyzed with one-way ANOVAs followed by post hoc Tukey-Kramer tests.

\section{Conflict of Interest}

The authors declare no conflict of interest.

Acknowledgements. This work was supported by a Grant-in-Aid for Scientific Research on Innovative Areas (25122711) from the Japan Society for the Promotion of Sciences to RM, a Grant-in-Aid from Ministry of Health, Labour and Welfare to RM, the Osaka University Program for the Support of Networking among Present and Future Researchers to RM, and a Grant-in-Aid for Scientific Research (S) from JSPS (25221309) to TY.

1. Smith KJ, Blakemore WF, McDonald WI. The restoration of conduction by central remyelination. Brain 1981; 104: 383-404.

2. Nave KA. Myelination and the trophic support of long axons. Nat Rev Neurosci 2010; 11: 275-283.

3. Achiron A, Barak Y. Multiple sclerosis-from probable to definite diagnosis: a 7-year prospective study. Arch Neurol 2000; 57: 974-979.

4. Prineas JW, Connell F. Remyelination in multiple sclerosis. Ann Neurol 1979; 5: 22-31.
5. Prineas JW, Barnard RO, Kwon EE, Sharer LR, Cho ES. Multiple sclerosis: remyelination of nascent lesions. Ann Neurol 1993; 33: 137-151.

6. Irvine KA, Blakemore WF. Remyelination protects axons from demyelination-associated axon degeneration. Brain 2008; 131: 1464-1477.

7. Franklin RJ. Why does remyelination fail in multiple sclerosis? Nat Rev Neurosci 2002; 3 : 705-714.

8. Dawson MRL, Polito A, Levine JM, Reynolds R. NG2-expressing glial progenitor cells: an abundant and widespread population of cycling cells in the adult rat CNS. Mol Cell Neurosci 2003; 24: 476-488.

9. Young KM, Psachoulia K, Tripathi RB, Dunn SJ, Cossell L, Attwell D et al. Oligodendrocyte dynamics in the healthy adult CNS: evidence for myelin remodeling. Neuron 2013; 77 : 873-885.

10. Franklin RJM, Ffrench-Constant C. Remyelination in the CNS: from biology to therapy. Nat Rev Neurosci 2008; 9: 839-855.

11. Lucchinetti C, Brück W, Parisi J, Scheithauer B, Rodriguez M, Lassmann H. A quantitative analysis of oligodendrocytes in multiple sclerosis lesions. A study of 113 cases. Brain 1999; 122: 2279-2295.

12. Schölkens BA, Bartmann W, Beck G, Lerch U, Konz E, Weithmann U. Vasodilation and inhibition of platelet aggregation by prostacyclins with modified omega-side chain. Prostaglandins Med 1979; 3: 7-22

13. Vane JR, Botting RM. Pharmacodynamic profile of prostacyclin. Am J Cardiol 1995; 75 : $3 \mathrm{~A}-10 \mathrm{~A}$.

14. Oida $\mathrm{H}$, Namba $\mathrm{T}$, Sugimoto $\mathrm{Y}$, Ushikubi $\mathrm{F}$, Ohishi $\mathrm{H}$, Ichikawa A et al. In situ hybridization studies of prostacyclin receptor mRNA expression in various mouse organs. $\mathrm{Br} \mathrm{J}$ Pharmacol 1995; 116: 2828-2837.

15. Siegle I, Klein T, Zou MH, Fritz P, Kömhoff M. Distribution and cellular localization of prostacyclin synthase in human brain. J Histochem Cytochem 2000; 48: 631-641.

16. Cazevieille C, Muller A, Bonne C. Prostacyclin (PGI2) protects rat cortical neurons in culture against hypoxia/reoxygenation and glutamate-induced injury. Neurosci Lett 1993; 160: $106-108$.

17. Fang YC, Wu JS, Chen JJ, Cheung WM, Tseng PH, Tam KB et al. Induction of prostacyclin/PGI2 synthase expression after cerebral ischemia-reperfusion. J Cereb Blood Flow Metab 2006; 26: 491-501.

18. Muramatsu R, Takahashi C, Miyake S, Fujimura H, Mochizuki H, Yamashita T. Angiogenesis induced by CNS inflammation promotes neuronal remodeling through vessel-derived prostacyclin. Nat Med 2012; 18: 1658-1664.

19. Howe AK. Regulation of actin-based cell migration by cAMP/PKA. Biochim Biophys Acta 2004; 1692: 159-174.

20. Hall SM. The effect of injections of lysophosphatidyl choline into white matter of the adult mouse spinal cord. J Cell Sci 1972; 10: 535-546.

21. Jeffery ND, Blakemore WF. Remyelination of mouse spinal cord axons demyelinated by local injection of lysolecithin. J Neurocytol 1995; 24: 775-781.

22. Murray PD, McGavern DB, Sathornsumetee S, Rodriguez M. Spontaneous remyelination following extensive demyelination is associated with improved neurological function in a viral model of multiple sclerosis. Brain 2001; 124: 1403-1416.

23. Jeffery ND, Blakemore WF. Locomotor deficits induced by experimental spinal cord demyelination are abolished by spontaneous remyelination. Brain 1997; 120: $27-37$.

24. D'Amelio P, Cristofaro MA, D'Amico L, Veneziano L, Roato I, Sassi F et al. lloprost modulates the immune response in systemic sclerosis. BMC Immunol 2010; 11: 62.

25. Jung S, Donhauser T, Toyka KV, Propentofylline Hartung H. and lloprost suppress the production of TNF- $\alpha$ by macrophages but fail to ameliorate experimental autoimmune encephalomyelitis in Lewis rats. J Autoimmun 1997; 10: 519-529.

26. Boswell MG, Zhou W, Newcomb DC, Peebles RS Jr. PGI2 as a regulator of CD4 + subset differentiation and function. Prostaglandins Other Lipid Mediat 2011; 96: $21-26$.

27. Dore-Duffy P, Donaldson JO, Koff T, Longo M, Perry W. Prostaglandin release in multiple sclerosis: correlation with disease activity. Neurology 1986; 36: 1587-1590.

28. Mirshafiey A, Jadidi-Niaragh F. Prostaglandins in pathogenesis and treatment of multiple sclerosis. Immunopharmacol Immunotoxicol 2010; 32: 543-554.

29. Marusic S, Leach MW, Pelker JW, Azoitei ML, Uozumi N, Cui J et al. Cytosolic phospholipase A2 $\alpha$-deficient mice are resistant to experimental autoimmune encephalomyelitis. J Exp Med 2005; 202: 841-851.

30. Ayoub SS, Wood EG, Hassan SU, Bolton C. Cyclooxygenase expression and prostaglandin levels in central nervous system tissues during the course of chronic relapsing experimental autoimmune encephalomyelitis (EAE). Inflamm Res 2011; 60: 919-928.

31. Kihara Y, Matsushita T, Kita Y, Uematsu U, Akira S, Kira J et al. Targeted lipidomics reveals mPGES-1-PGE2 as a therapeutic target for multiple sclerosis. Proc Natl Acad Sci USA 2009; 106: 21807-21812.

32. Lin X, Ramamurthy SK, Le Breton GC. Thromboxane A2 receptor-mediated cell proliferation, survival and gene expression in oligodendrocyte. J Neurochem 2005; 90: $257-268$.

33. Sun X, Liu Y, Liu B, Xiao Z, Zhang L. Rolipram promotes remyelination possibly via MEKERK signal pathway in cuprizone-induced demyelination mouse. Exp Neuro 2012; 237: 304-311. 
34. Pearse DD, Pereira FC, Marcillo AE, Bates ML, Berrocal YA, Filbin MT et al. CAMP and Schwann cells promote axonal growth and functional recovery after spinal cord injury. Nat Med 2004; 10: 610-616.

35. Raible DW, McMorris FA. Cyclic AMP regulates the rate of differentiation of oligodendrocytes without changing the lineage commitment of their progenitors. Dev Biol 1989; 133: 437-446.

36. Sato-Bigbee C, DeVries GH. Treatment of oligodendrocytes with antisense deoxyoligonucleotide directed against CREB mRNA: effect on the cyclic AMP-dependent induction of myelin basic protein expression. J Neurosci Res 1996; 46: 98-107.

37. Raible DW, McMorris FA. Oligodendrocyte differentiation and progenitor cell proliferation are independently regulated by cyclic AMP. J Neurosci Res 1993; 34: 287-294.

38. McMorris FA, Furlanetto RW, Mozell RL, Carson MJ, Raible DW. Regulation of oligodendrocyte development by insulin-like growth factors and cyclic nucleotides. Ann N Y Acad Sci 1990; 605: 101-109.

39. Murata T, Ushikubi F, Matsuoka T, Hirata M, Yamasaki A, Sugimoto Y et al. Altered pain perception and inflammatory response in mice lacking prostacyclin receptor. Nature 1997 388: $678-682$.

40. ladecola C, Nedergaard M. Glial regulation of the cerebral microvasculature. Nat Neurosci 2007; 10: 1369-1376.
41. Lok J, Gupta P, Guo S, Kim WJ, Whalen MJ, van Leyen K et al. Cell-cell signaling in the neurovascular unit. Neurochem Res 2007; 32: 2032-2045.

42. Guo S, Lo EH. Dysfunctional cell-cell signaling in the neurovascular unit as a paradigm for central nervous system disease. Stroke 2009; 40: S4-S7.

43. Arai K, Lo EH. An oligovascular niche: cerebral endothelial cells promote the survival and proliferation of oligodendrocyte precursor cells. J Neurosci 2009; 29: 4351-4355.

44. Basso DM, Fisher LC, Anderson AJ, Jakeman LB, McTigue DM, Popovich PG. Basso Mouse Scale for locomotion detects differences in recovery after spinal cord injury in five common mouse strains. J Neurotrauma 2006; 23: 635-659.

(i) (5) (2) Cell Death and Disease is an open-access journal published by Nature Publishing Group. This work is licensed under a Creative Commons Attribution-NonCommercialShareAlike 3.0 Unported License. To view a copy of this license, visit http://creativecommons.org/licenses/by-nc-sa/3.0/ 